\title{
The Network Level Needed in Determining Organizational Structure
}

\author{
Altin Kavadarli \\ Bogazici University, Istanbul, Turkey \\ kavadarli@superonline.com
}

\begin{abstract}
As the external operations of organizationsbecome more complex with increasing globalization and as structures encounter difficulties in multi-tasking and increased information flow management, there is a need for more flexible and faster way of organizing. In order to promote efficiency and flexibility, organizations' restructuring efforts result from need for coordination and work that increasingly occurs through informal networks of relationships rather than through formal structures. As the rapid adoption of social media and mobile computing are transforming how organizations in all industries relate to their environment, organizationalnetworks can be found inside every business and shape how employees share, communicate and collaborate at the workplace. New structures are emerging that blend different types of networks and hierarchies. The nature and complexity of the business determines the best the way its organizational structure is organized and this paper presents a theoretical model to help determine ideal organizational structures for different needs in order to deal with the pace and ambiguity of the business environment.
\end{abstract}

Keywords: Network organization, Organizational structure, Strategic management, Hierarchy

Network Organizations Definition: An organization is an entity, such as an institution or an association, that has a collective goal and is linked to an external environment. A network organization is a group of legally independent companies or subsidiary business units that use various methods of coordinating and controlling their interaction in order to function as a larger entity. A network organization is a new pattern emerging in many progressive 21st century enterprises. A social network consists of a set of actors ("nodes") and the relations ("ties" or "edges") between these actors (Wasserman\& Faust, 1994). A business network meanwhile, is a set of two or more connected business relationships, in which each exchange relation is between business firms that are conceptualized as collective actors (Emerson, 1981). In other words, it refers to relationships between multiple firms that interact with each other (Möller and Wilson, 1995). Networks are constructed when individuals, whether organizations or humans, interact. This implies new ways of working, with consequences for the organization's structure, processes, people and culture. A network organization is a collection of autonomous firms or units that behave as a single larger entity, using social mechanisms for coordination and control.

\section{Introduction}

Traditional organizations integrate work vertically, where they place authority in a pyramidal, hierarchical structure. In the pyramid shape, power is concentrated primarily at the top. This hierarchical organizational form first emerged in the United States in the late 19th century with the arrival of mass production. Hierarchy eliminates anomaly, standardizes processes, solves short-term problems, and achieves efficiency with its mode of operating. The hierarchical organizationwasan incredible invention that could direct and coordinate the actions of many people that produce and sellvarious products or services tofar away distancesand do so effectively, efficiently, and profitably, time after time. However, hierarchy and its managerial processes do not handle transformation well and in a world with an ever-increasing rate of change, it is impossible to succeed without fast transformations. The problem with hierarchy is that its management processes hinder change, because they operate on autopilot, where everyone is ideally situated within an unthinking and predictable machine. Thus, hierarchy ignores new opportunities that require transformation because these do not go parallel with its core focus on maintenance and optimization. Hierarchies are structures consisting of internal networks, in which external network systems are almost not present,however, in the recent years, significant restructuring efforts have resulted in organizations with fewer hierarchical levels and more elastic functional and organizational boundaries.

This paper aims to explore these new structures and present a theoretical model to determine what kind of structures are needed to deal with change and uncertainty. In order to promote efficiency and flexibility, 
these restructuring efforts requirecoordination and work that increasingly occurs through informal networks of relationships rather than through formal structures or work processes. By establishing external networks, theserelationships become central to performance and strategy execution. Organizational network analysis can provide an understanding to the inner workings of an organization as networks are means of making invisible patterns of information flow and means of making collaboration in strategically placed groups. As organizations restructure to respond to their everchanging environments, there has been a growing need for new kinds of organizational structures. The "Networked Organization" is one such response in which Lipnack and Stamps (1999) define it as one: "Where independent people and groups act as independent nodes, link across boundaries, to work together for a common purpose; it has multiple leaders, lots of voluntary links and interacting levels. "These new ways of organizing show characteristics of innovation, resilience, and selfmanagement and havebeen described as the lattice organization, the spider's web, the holonic enterprise, and the virtual corporation. All describe new ways of organizing where:authority is not through hierarchy; people and teams are linked across conventional boundaries (ie. departments, geographies); members and structurescan fastly adapt to everchanging circumstances; management style is through mutual responsibility and sharing; new ways to work effectively are explored; and teams are adjusted or dissolved as needed.

\section{Literature Review}

"Organizations are and always will be run by people. They are infused with purpose and meaning only through the imagination and will of people..." (Lawrence and Lorsch, 1967) The network highlights the importance of the social side of people's actions in organizational settings where the focus is not only on optimality or efficiency but also on interconnectedness. "We know that personal interaction patterns in organizations are associated with power, turnover, information flows, attitudes, promotion opportunities, and social support" (Salancik, 1995). Network analysis (social network theory) is the study of how the social structure of relationships around a person, group, or organization affects beliefs or behaviors. Pressure is inherent in a social structure and network analysis is a set of methods for detecting and measuring the magnitude of the pressures. The focus of the network approach is that reality should be primarily conceived and investigated from the view of the properties of relations between and within units instead of the properties of these units themselves, as it is a relational approach. In social and communication science these units are social units such as individuals, groups, organizations and societies.

Rogers characterizes a communication network as consisting of "interconnected individuals who are linked by patterned communication flows" (Rogers, 1987). While the original focus of network research was on understanding how the embeddedness of individuals influences their behavior, a similar approachwas applied to organizations (Burt, 1982; Walker, 1988; Mizruchi, 1992; Gulati, 1998). A communication network analysis studies "the interpersonal linkages created by the shearing of information in the interpersonal communication structure," (Rogers, 1987) that is, the network. Network scholars have focused on the role of the social context, primarily, the cumulation of prior ties between firms for the formation of new organizational networks (Gulati, 1995; Gulati and Gargiulo, 1999). The term "network" refers to a set of nodes and the relationships that connect them (Fombrun, 1982). The notion of a network implies nodes and links. The nodes can be people, teams or even organizations as networks operate at many levels and the links are the various coordination and adjustment mechanisms. Two way links and reciprocity across the links is what makes networks work.The following terms are used in order to describe a social network (Tichy et al., 1979).

The size of the network is important, just as the density (or connectedness), which is the number of links in a network as a ratio of the total possible links. The degree of clustering determines the situation where dense regions of interconnected individuals appear in parts of the network. The term openness is used to describe the ratio of external links against the total number of possible external links. The term stability is used to explain the need for changes or lack of changes in the networks over time. Reachability is a measure of thenumber of links between any two nodes. Finally, centrality is the degree to which the formal hierarchy guides relations in the network.In a network, where formal authority and communications in hierarchical organizations often fail, high degrees of informal communications succeed. Network organizations are characterized by autonomy maintenance, relationship building, power sharing, and influence processes. They are open systems that respond to environmental circumstances and exhibit multiple levels of compexities as 
they constantly engage in integration mechanisms to penetrate their boundaries. The benefits of the networked organization are: being close to the customer; maximizing the knowledge potential of an organization; minimizing disfuntion; and responsiveness and adaptiveness.

Network organizations are defined by elements of structure, process, and purpose. The design elements of cospecialized assets, joint control, and collective purpose distinguish network organizations from centralized organizations and inflexible hierarchies. (Van Alstyne,1997) Network organizations combine co-specialized and possibly intangible assets under shared control in their structure and joint ownership is essential in order to achieve an integration of assets and intercommunication in an efficient and flexible manner. As the external operations of organization become more complex with increasing globalization and as structures encounter difficulties in multi-tasking and increased information flow management, there is a need for organizational change and innovation in order to meet the increasing challenges. Network organizations can be seen as a blend of market and firm and they are somewhere between vertical integration and market disaggregation. Network organizations are more like firms than markets, but they occur in situations where demand uncertainty makes vertically integrated firms a bad idea. The conditions that favor network organizations are: Frequent Transactions, Demand Uncertainity, Customization, Task Complexity, and Structural Embeddedness. Most of these conditions are the same as those favoring firms rather than markets. A network organization is like an ordinary firm which does not have a system of direct supervision, nor standardized rules and procedures that apply throughout the firm. Consequently, they have to coordinate and control the units in some other way and some of the ways they do this are: Joint Payoffs, Restricted Access, Reputation, and Macroculture.

By examining today's most forward-looking organizations, we can identify the most important features of network organizations as; Borderless, Collaborative, and Pervasively-networked. Rockartand and Short (1991) listed the dimensions of the networks as:

- Shared goals as a necessary condition for organization of activities;

- Shared knowledge expertise an underlining force for effectiveness;

- Shared work as a means for incorporation of groups outside of local structure;

- Shared decision-making as a means for introduction of expertise from across the organizational spectrum;

- Shared timing and issue prioritization as a means for enhancing the action stepsparticularly in response to critical issues;

- Shared responsibility, accountability and trust as elements further strengthening the intraorganizational cohesion; and

- Shared recognition and reward as implicit in the effective functioning of networks

\section{Position of the Paper}

"Network analysis corrects a tendency in organizational theory to focus on the trees rather than the forest, on the actions of individual organizations rather than on the organization of their actions" (Salancik, 1995). A fully networked organization works through flexible networks and skills to get things done. This is a theoretical idea in some ways, as all organizations have and need some form of structure, but it reflects a desire towards flexibility and away from a formal system of reporting and solid structure. This more flexible and fast changing way of organizing requires a high degree of elasticity and skills in order to deal with this significant increase in complexity and ambiguity. Ambidexterity is needed since leadership, collaboration and personal effectiveness become different in this more flexible way of organizing. Organizations need to develop a 'softer structure' that networked organizations require in order to succeed and these 'softer' ways of working need to be established, managed and supported in different ways.

Networked organizations are a result of the need for organizations to work across the traditional vertical structure of function and geography, without a horizontal structure to replace the vertical. In a network organization, teams and groups can quickly come together around a particular activity and utilize the capability and resources they need from across the whole organization. Projects, teams and workflow cut 
across functions and geography and teams can quickly be formed to meet a particular business need and get dissolved as quickly when the business is done. As decision-making members intervene and extend their influence through association, they alter the resources for themselves, their networks, and their competitors. Through this process the structure of even the network itself can change (Van Alstyne,1997). A network organization assumes a unifying purpose and the need for a sense of identity in managing the resources, parties, and actions necessary for conducting the strategy and goals of purpose. The rapid adoption of social media and mobile computing are transforming how organizations in all industries relate to their environment. Organization networks can be found inside every business and shape how employees share, communicate and collaborate at the workplace. The rise of the networked organization poses great opportunities for businesses, while changing the construct of leadership to break through organizational chaos and develop a new corporate culture, ethics and leadership skills. It is this kind of cultural change paradigm that will probably define the networked organizations of the future.

Organizations today need to respond more swiftly as cooperative problem solvers to their increasingly complex environments in the face of rising competition and change. Competition and change together increase problem complexity by increasing the number of dynamic variables that organizaitons must consider. The more rapid the change, the greater the decrease in the acceptable response time. The need for speed and expertise justifies the movement to points of execution, but it is inevitable some miscommunication and delays occur. The ability to interpret ambiguous circumstances is inherent and human-specific asknow-how cannot be easily transferred to or centralized in a single decision maker. Change not only creates complexity, but it also creates risk because intervening in the environment to control various externatilities can prove both ineffective and costly if partners are not interconnected. Change also cannot be managed without trust or open and efficient communicationbetween partners. Combining these perspectives results in the interpretation of network organizations as integrated systems with co-specialized assets and joint control.In summary, the primary characteristic of the networked organization comprise of:

- Shared goals reinforced through the mechanisms of social networks and informal relations as well as trust

- Support of information technologies comprising a basis for connection and information flows that essentially bind and maintain the organization

- Low hierarchy and centralization, with managerial responsibilities shared and overlapping across the organization

- The organizational integration over vertical and horizontal boundaries

The variety of features characterizing network organization integrates all these perspectives to helpparticipants address rising complexity as cooperative problem solvers. Knowledge sources are important to solving large scale integrative problems and agents are indispensable in their expertise by working in groups. To function well as a group, mutually agreeable goals should be established as well as a coherent group identity, and norms for action and exchange that enable and disable group and non-group actions.With a network structure, knowledge workers are able to create and leverage information to increase competitive advantage through the collaboration of small and adaptable self-directed teams. In order for this to happen, the organizational culture needs to change from a hierarchial to an agile structure enabling multiple forms of organizing within the same organization.Many studies show that synergy helps motivate agents to work together and grouping requires agents to balance several needs: flexibility and stability, generalization and specialization, and decentralization and centralization.

\section{Theoretical Model}

Organizational ambidexterity refers to an organization's ability to be efficient in its management of today's business and also adaptable for coping with tomorrow's changing demand. Organizational ambidexterity requires the organizations to use both explorationand exploitation techniques to be successful, integrating both internal and external network systems. Most probably, the successful organization of the future will have a combined organizational structure of hierarchies, where only internal networks exist,as well as more elastic and adaptive structures consisting of external networks. Both structures should be designed and integrated for a common purpose, since, while the hierarchy is still as important as it has always been for work optimization, the network organization is where the big changes can occur, allowing an organization to 
spot opportunities faster and then transform itself to utilize them. A network organization is a system of teams with individuals from all divisions and all levels, who leave formalities to participate in a nonhierarchical structure. The need for change in the environment should determine the balance an organization strives to achieve between hierarchy where internal networks are used and external network systems. Due to the vast potentially different combination of the employees' formal hierarchical and informal community participation, each organization is a unique type along a spectrum between a pure hierarchy and a pure community (flat) organizational structure. (Lim, Griffiths, and Sambrook, 2010).

The hierarchical structure should be used to manage production processes like automobile assembly where production could be broken down into a series of simple steps. Hierarchycontrols and manages all activities of a business from the raw materials to their allocation to consumers with internal networks. A centralized managerial hierarchy controls the entire production process, with mid-level managers establishing rules and procedures to manage a widebottom-line workforce.Hierarchy with only internal networks is efficient and effective when the complexity of business as well as the need for change is low. Until the early 1980's, the trend was to build increasing layers of management with more staff specialists, as this centralized hierarchical structure was seen as effective for managing large number of workers, but it lacked agility and was unable to process information rapidly throughout the organization.Research argues that as commerce becomes more global, hypercompetitive and turbulent, both markets and hierarchies displayed inefficiencies as modes of organizing production (Miles \& Snow, 1992; Powell, 1990) As business became more complex, these rigid structures grew increasingly untenable and so management theorists began to look for other ways. The model proposed below is one matrix to deal with change and complexity as the main objective of an organizational structure is to support and complement the strategy of any business arrangement used to realize the objectives and goals of the organization.

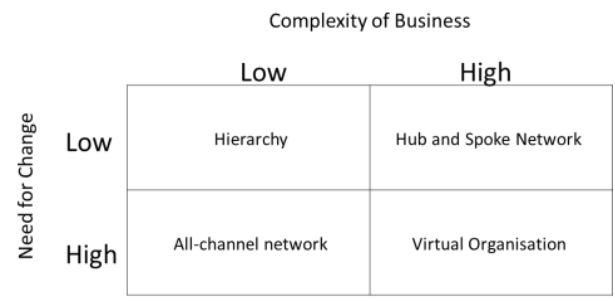

As the use of only internal networks was not effective enough in their adaptability to change, structures involving more external networks had to be established. As social capital theory suggests, a firm's external networks play a major role in contributing to its performance (Leenders \& Gabbay, 1999). Since the 1980's, many organizations have flattened their structures by shifting authority downward, giving employees increased autonomy and decision-making power. Advantages of flatter organization forms are in the decreased need for supervisors and middle management, faster decision making, and the ability to process information faster because of the reduced number of layers in the organization. Research shows that interconnectivity of networks in organizations can have a substantial impact on performance, learning, and innovation, and many benefits occur from well-connected networks between organizations (Smith, 1999). Together, competition and change increase problem complexity by increasing the number of dynamic variables that managers must consider while more rapid change decreases acceptable response time (Lawrence and Lorsch, 1967). A consequence of the types of flatter type of organizations, though, is that employees tend to be more dispersed both geographically and organizationally. To solve this problem, many organizations have eliminated superfluous processes and begun focusing on their core, value-creating business. Flat organizations using joint ventures and strategic alliances are providing increased flexibility and innovation, and are replacing many traditional hierarchies.

Rising competition and change cause organizations to respond more swiftly as cooperative problem solvers to their increasingly complex environments (Gasser and Hill, 1990). When the complexity of business is high (especially in the technological aspect), but the need for change is low, a more adaptable structure, which is the hub and spoke network seemsto be the best organizational structure.A hub and spoke network consists of centralized resources that support the various business units. The business units still have the freedom and 
flexibility to connect with the market, but are still in alignment with what other spokes are doing. This structure is often used in used in transport, telecommunications, and freight industries. THY (Turkish Airlines) and UPS (United Parcel Service) are two examples of organizations who use such a structure. Though the central unit is not necessarily the leader of the network, information passes through this element before reaching the other nodes. In contrast, when the complexity of business is low, but the need for change is high, an all-channel network system seems the best organizational structure to work in. In the all-channel, there is more control and discipline compared to the hub and spoke network. Hub and spoke network is more centralized with independent nodes, whereas the all-channel network relies on the links between nodes. Allchannel networks consist of individual units and every unit connects to every other unit as the network permits all members to communicate actively with each other. Although an all-channel network often features a central unit, or even multiple leaders, it is generally a collaborative system where units use decentralized decision-making processes, allowing for the autonomy of various local nodes. In this network, each node links to the next in sequence as information passes down a single line.

The all-channel model is becoming increasingly significant as a source of organizational collaborative power, withcriminal organizations as the most frequently cited examples. The all-channel network has no central leadership and no key node whose removal can disrupt the entire organization. Instead, the network is completely decentralized, allowing for indivudual initiative and autonomy in an organization. The all-channel network is one of the most difficult to maintain because it requires very strong communication to maintain ties between nodes, so this form of organizations have only recently become feasible with the coming of the information age. The paradigm shift to new organizational structuresis a response to unprecedented customer expectations and alternatives, global competition, time compression, complexity, rapid change, and increased use of technology. When both the complexity of business (especially in the technological aspect) and need for change is high, the concept of virtual organization appears in the picture. A virtual organization is a network of companies which support each other around a product and or a service idea. Virtual organization is an alliance of companies formed for the purpose of delivering specific products and/or services. The company who is responsible for the products and/or servicesis sometimes called the core company. The companies in the network are tightly integrated by information and communication technologies so that it is not apparent that the different processes are handled by separate companies. The core company is linked together by information and communication technologies with other companies,sometimes called satellite companies.

Lipnack and Stamps (1999) have identified elements of virtuality in teams and organizations. They define a virtual team as "a group of people who interact through interdependent tasks guidedby common purpose that works across space, time, and organizational boundaries with linksstrengthened by webs of communication technologies. "They offer three key features for a successful virtual team. One is the choice of team members with the appropriate skills and knowledge for the task, second is the definition of a purpose to steer the group, and third is the effective linking of team members, including communication channels, interactions, and relationships. The most common examples given for virtual organizations are the Hollywood film industry and British Telecom. These new virtual organizations consist of groups and individuals from different companies that might include customers, competitors, and suppliers who have a focused purpose of bringing a product/service to market as rapidly as possible. As organizations create these many new linkages, advanced information technology becomes an important aspect and the key to the success of a virtual organization.

Virtual network structures bring together different companies with the aim of obtaining and maintaining a competitive advantage over others outside of their network. With the availability of internet and other communication technologies, a virtual organization is an option for many innovative entrepreneurs. An organization can theoretically span across the entire globe with the availability and infinite nature of networked based information technology. The characteristics of these organizations include a purpose that is motivated by specific market opportunities, world-class core competence, information networks, interdependent relationships, and permeable boundaries. Virtual organizations represent structures that are motivated by specific market opportunities and once the alliance has been formed and the opportunity has been exploited, partners may move on to new partnerships and alliances. Each partner in a virtual corporation contributes a world-class core competence, such as production, design, manufacturing, or 
marketing and this ability of multiple firms to create synergies among functions and processes creates many unexplored possibilities. Virtual organizations can be considered as the newly emerging elastic and adaptable organizational structure.

\section{Conclusion}

Most of today's organizations were built on Weber's principles of bureaucratic management theory, including structuring an organization into a hierarchy and having clearly defined rules to help govern an organization.Now, however, there is a shift from hierarchical to networked organizations. The use and popularity of the network concept by social science researchers intensified in recent years, as scholars started to recognize the relevance and importance of relations for business activity (Easton \& Araujo, 1986; Harland, 1995; Nohria \& Eccles, 1992). This paper has examined the network organization literature to explain its multiple variables.The extremely bureaucratic organization structure of hierarchies, with only internal networks, is stillconsidered adequate to manage a vast number of employees, however the needs of today's business world create the need for organizations that can handle change better and faster. In our globalizing world, organizations have to deal with an increasingly dynamic work environment that require continuous adaptation to a variety of changes in the operations. The new business climate is characterized by profound and continuous changes due to globalization, exponential leaps in technological capabilities, and other market forces. These changes have brought on the network organizations, which are defined by highly co-specialized assets, an integrative joint control, and common purpose which tend to perform well under conditions which require both efficiency and flexibility. The form that emerged as the result, thenetwork organizational form balanced the flexibility of markets with the predictability of traditional hierarchies (Achrol, 1997; Miles \& Snow, 1992; Powell, 1990; Snow, Miles \& Coleman, 1992)

A 'business network' as defined by Yeung (1994, p. 476) is "an integrated and coordinated set of ongoing economic and non-economic relations embedded within among and outside business firms". Network organizations tend to perform well under conditions which require both efficiency and flexibility. Literature emphasizes that information and know-how are important network organization assets. Knowledge capital creates value and information links increase the ability to handle complexity. As the environment changes in various ways, this new system senses and responds to it, and in turn creates more solutions to adapt to a larger change with external network webs. Network organizations identify potential chances, anticipate future possibilities, establish strategies for organization-wide transformations, recognize and address barriers, and finally achieve change. The advantage of network organizational structure over any other form of organizational structure is its flexibility and efficiency. A whole new system that is much bigger, more powerful and that involves far more people is formed.

In the recent years, many high-performing organizations use structures with external networks, rather than traditional hierarchy of only internal networks, in order to lead and manage change, even going as far as establishing of virtual organizations.The nature and complexity of the business determines best the way its organizational structure is organized. The networked configuration of work systems affords intercommunication among differentially organized areas of work. Rapid developments of information and communications technology is tranforming the industrial age to the information age and the network organization seems a sensible response to the complex environment of the new millenium business. In this world of rapid change and uncertainty, organizations need to continually renew, revitalize and reinvent themselves in order to respond fast and creatively. The network-centric approach taps into the hidden resources of knowledge workers supported and enabled by information and communications technology, although it must be noted that a network-centric organization is more about people and culture than technology. The theoratical model proposed in this paper is one to help determine organizational structures in order to deal with the pace and ambiguity of the business environment.

\section{References}

Achrol, R. S. (1997). Changes in the theory of inter-organizational relations: Toward a network paradigm. Journal of the Academy of Marketing Science, 25(1), 56-71

Burt, K. (1982). Towards A Stuctural Theory of Action. New York: Academic Press. 
Easton, G. \& Araujo, L. (1986). Networks, bonding and relationships in industrial markets. Industrial Marketing and Purchasing, 1(1), 8-25.

Emerson, R. M. (1981). Social exchange theory. Social psychology: sociological perspectives. New York: Basic Books. Entrepreneurship and Regional Development, 12(3), 195-216.

Fombrun, C. J. (1982). Strategies for network research in organizations. Academy of Management Review, 7(2), 280-291.

Gasser, L. \& Hill Jr. R. W. (1990). Coordinated problem solvers. Annual Review of Computer Science, 4(1), 203253.

Gulati, R. (1998). Alliances and networks. Strategic Mnr agemmt Journal, 19, 293-3 17.

Gulati, R. (1995). Social structure and alliance formation pattern: A longitudinal analysis. Administrative Science Quarterly, 49, 61 9-52.

Gulati, R. \& Gargiulo, M. (1999). Where do interorganizational networks come from? Atnerican Journal of Sociology, 104, 193 9-9 3.

Harland, C. M. (1995). Networks and globalisation-A review of research. EPSRC Final Report.

Lawrence, P. R. \& Lorsch, J. W. (1967). Differentiation and integration in complex organizations. Administrative science quarterly, 7, 1-47.

Lim, M., Griffiths, G. \& Sambrook, S. (2010). Organizational structure for the twenty-first century. annual meeting of The Institute for Operations Research and The Management Sciences, Austin.

Lipnack, J. \& Stamps, J. (1999). Virtual teams: The new way to work. Strategy \& Leadership, 27(1), 14-19.

Miles, R. E. \& Snow, C. C. (1992). Causes of failure in network organizations. California Management Review, $34,52-72$.

Mizruchi, M. S. (1992). The structure of corporate political action: Interfirm relations and their consequences. Harvard University Press.

Möller, K. K. \& Wilson, D. T. (1995). eds. Business marketing: An interaction and network perspective. Springer Science \& Business Media.

Nohria, N. \& Eccles, R. G. (1992). Networks and organizations: Structure, form, and action.

Powell, W. W. (1990). Neither market nor hierarchy: Network forms of organization. Research on Organizational Behavior, 12, 295-336

Rockartand, J. F. \& Short, J. E. (1991). The networked organization and the management of interdependence. The corporation of the 1990s: information technology and organizational transformation, 189.

Rogers, E. M. (1987). Progress, problems and prospects for network research: Investigating relationships in the age of electronic communication technologies. Social Networks, 9(4), 285-310.

Salancik, G. R. (1995). Wanted: A good network theory of organization, 345-349.

Smith, O. J. (1999). Network position and firm performance. Andrews S, Knoke D. Research in the Sociology of Organizations Greenwich, Conn: JAI Press, 16, 129-159.

Snow, C. C., Miles, R. E. \& Coleman, H. J. (1992). Managing 21st century organizations. Organizational Dynamics, 20(3), 5-20

Tichy, N. M., Tushman, M. L. \& Fombrun, C. (1979). Social network analysis for organizations. Academy of management review, 4(4), 507-519.

Van Alstyne, M. (1997). The state of network organization: a survey in three frameworks. Journal of Organizational Computing and Electronic Commerce, 7(2-3), 83-151.

Yeung, H. W. (1994). Critical reviews of geographical perspectives on business organizations and the organization of production: towards a network approach. Progress in Human Geography, 18(4), 460490.

Walker, G. (1988). Network analysis for cooperative interfirm relationships. in F. K. Contractor and P. Lorange (eds), Cooperative Strategies in International Business, Lexington, KY: Lexington Press. 227-40.

Wasserman, S. \& Katherine, F. (1994). Social network analysis: Methods and applications, 8. Cambridge university press, 1994. 